\title{
Synthesis and quality control of viral membrane proteins
}

\author{
Claudia MagGioni AND InEKe BRAaKMAN \\ University of Utrecht, Padualaan 8, 3584 CH Utrecht, The Netherlands \\ e mail: \\ I.Braakman@chem.uu.nl \\ telephone:+31-30-2532759
}

CONTENTS:

6.1 Protein biosynthesis

6.1.1 Entering the ER: targeting and translocation

6.1.2 Protein folding in the ER

Chaperones and folding enzymes

Glycosylation and the CNX/CRT cycle

Disulfide bond formation

6.2 Quality control

6.2.1 Retention in the ER

6.2.2 Degradation and the unfolded protein response

6.3 How viruses evolve to fool the host 
The envelopes of virus particles contain one or more types of integral membrane proteins. The most abundant ones contain a single transmembrane domain, a large ectodomain that is localized at the outside of the viral envelope, and a small cytoplasmic domain. Almost all viral membrane proteins are oligomeric, either homooligomeric or heterooligomeric. Each subunit consists of one or two polypeptide chains which can be held together by noncovalent interactions or by covalent disulfide bridges. In case of two polypeptide chains, they often are derived from a single chain precursor which is cleaved proteolytically during transport to the plasma membrane. The cleavage is accompanied by conformational changes that activate biological activity. For most of the viral proteins the cleavage is performed in trans by cellular or viral proteases. An exception is the capsid protein of the alphavirus genus, which, being a serine protease, cleaves itself immediately after synthesis in the ER (Melancon and Garoff 1987).

Viral envelope proteins have different functions: they mediate binding to the receptor(s) on the host cell's plasma membrane, membrane fusion and penetration. For some viruses all these functions are combined in a single glycoprotein. In addition, some small viral envelope proteins have been shown to possess ion channel activity (reviewed by Fischer and Sansom 2002). The channel protein M2 of Influenza A for example possesses proton conductance activity and is responsible for the change of $\mathrm{pH}$ in the interior of the virion that is necessary for disassembly of the virus particle. This class of viral membrane proteins contains one or more transmembrane domains and either lacks or has a lower number of glycans and disulfide bonds.

To be functional, as is true for all proteins, viral envelope (glyco)proteins must reach the correct three-dimensional structure, and this process is referred to as protein folding. To translate and fold viral envelope proteins, viruses use the host cellular machinery and they are subjected to the same quality control systems as endogenous proteins. 


\subsection{Protein biosynthesis}

\subsubsection{Entering the endoplasmic reticulum: targeting and translocation}

The biogenesis of most secretory and membrane proteins involves targeting of the nascent protein to the endoplasmic reticulum (ER), translocation across or integration into the ER membrane, and maturation into a functional product. Leader peptides of nascent chains are recognized in the cytosol by signal recognition particle (SRP) as soon as they emerge from the ribosome. The SRPnascent chain-ribosome complex interacts with the SRP receptor which targets it to the ER. Once targeted, the nascent chain is transferred to the translocation channel in a GTP-dependent step. The ribosome-nascent chain complex is tightly bound to the translocon in a way that the ER lumenal and cytosolic contents are not in communication. The translocation channel is the heterotrimeric Sec61p complex, composed of $\square, \square$, and $\square$ subunit (reviewed by Matlack et al. 1998). The channel is not a passive hole but is a dynamic structure that accomplishes different functions including targeting, co-translational translocation and cotranslational integration (Johnson and van Waes 1999).

Other proteins that are part of the translocation machinery are the transloconassociated protein (TRAP) complex with unknown function (Hartmann et al. 1993; Wang and Dobberstein 1999), the translocating chain associating membrane protein (TRAM), the small ribosome-associated membrane protein 4 (RAMP4) (Gorlich and Rapoport 1993), the signal peptidase (SP) and oligosaccharyl transferase (OST). TRAM is necessary for translocation of most substrates and regulates which domains of the nascent chain are "accessible" from the cytosol during a translocational pause (Hegde et al. 1998). Signal peptidase is necessary for cleavage of leader peptides and the OST for transfer of oligosaccharide chains to nascent proteins, two processes that as a rule occur co-transl(oc)ationally. Exceptions to this rule were found for some viral proteins. The leader peptides of HIV-1 Envelope (Berman et al. 1988; Li et al. 1994; Land et al. 2003) and the signal peptide of a portion of the newly sythesised hCMV US11 (Rehm et al. 2001) were removed only after synthesis of these proteins was completed. 
Glycosylation of the hepatitis C virus envelope protein E1 can occur also posttranslationally albeit in a mannosylphosphoryldolichol-deficient $\mathrm{CHO}$ mutant cell (Duvet et al. 2002). Because molecular details of biosynthetic processes are more frequently studied in viral proteins than mammalian proteins, we can only speculate on the specificity of these exceptions for viral proteins, and on the abundance of them in both the viral and mammalian world.

Not only the translocon and the associated proteins, but also ER lumenal proteins have been shown to be necessary for proper translocation. For example BiP, the Hsp70 homolog in the ER, was found to be responsible for sealing the lumenal end of not only the ribosome-free but also the ribosome-docked translocon (Haigh and Johnson 2002).

\subsubsection{Protein folding in the ER}

The ER is the entry point for newly synthesised proteins that are directed into the secretory pathway. Folding can occur spontaneously because all information needed to reach the proper three-dimensional structure is present in the primary sequence (Anfinsen 1973). Still, within the cell the process needs assistance, which is provided by helper proteins known as molecular chaperones and folding enzymes. The ER is specialized for protein folding, providing an optimized environment: oxidising conditions and a high concentration of chaperones and folding enzymes. Folding begins co-translationally and continues for minutes to hours after termination of polypeptide synthesis.

Some viral glycoproteins not only need co-translational folding but also cotranslational assembly in order to reach their proper, native structure. For example, correct folding of the envelope glycoprotein E1 of Hepatitis C virus, requires the presence of E2 (Michalak et al. 1997) through an interaction between the transmembrane domains of the two proteins (Cocquerel et al. 2001; Patel et al. 2001). In addition, another flanking protein of E1, the core protein, seems to play a role for correct folding of E1 (Merola et al. 2001). The folding process of p62 and E1, the envelope glycoproteins of Semliki Forest virus, is coordinated as well. The p62 protein can efficiently fold without E1, but E1 is found in aggregates in 
the absence of p62 (Andersson et al. 1997). Sindbis, another alphavirus, has an envelope composed of 80 trimers of E1-E2 dimers. The precursor of E2, pE2, dimerizes with E1 but, in this case, p62 interaction is needed for assembly and exit from the ER rather than for proper folding of E1 (Carleton et al. 1997). A recent study on the flavivirus tick-borne encephalitis (TBE) virus (Lorenz et al. 2002) clearly shows perhaps the first example of a mutual need for proper folding of the two envelope glycoproteins $\mathrm{E}$ and the precursor of $\mathrm{M}$ (prM), suggesting a chaperone-like function for prM. At the same time, E is also necessary for rapid signal sequence cleavage of prM and the two proteins can be each other's helpers even when expressed in trans, from different constructs.

\section{Chaperones and folding enzymes}

The main challenge a newly synthesized protein encounters within a cell is the intracellular environment, in particular the extremely high protein concentration of more than $200 \mathrm{mg} / \mathrm{ml}$ (Ellis 2001). A nascent chain is prone to aggregation and misfolding because of this high concentration (molecular crowding) and because of the close proximity of other nascent polypeptides emerging from a polyribosome. Considering that ribosomes on the same mRNA were found at a distance of only 80 nucleotides (Hesketh and Pryme 1991), or even 27 nucleotides during ribosome pausing (Wolin and Walter 1988), nascent chains in the lumen of the ER should emerge at a distance of about 15-40 nm, corresponding to a chain length of only a hundred amino acid residues or less. In the living cell, nonproductive protein folding generally is prevented by the action of molecular chaperones. Two types of chaperones can be recognized: the general kind, with a highly promiscuous interaction pattern, and the private kind, which caters one particular protein (family) only.

\section{Figure}

In the ER, these general chaperones, present also at high concentration (Fig 1A), associate with the growing nascent chain during translocation and continue to assist folding until a protein has acquired its native structure. Molecular chaperones act by facilitating rate-limiting steps, by stabilising unfolded proteins and by preventing undesired inter- and intra-chain interactions that could lead to 
aggregation. They may recognize hydrophobic surface patches, mobile loops and lack of compactness, or they may recognize a specific amino acid sequence in case of the private chaperones. These features are exposed transiently during folding, resulting in only transient associations with ER folding factors if the newly synthesized protein folds correctly.

The general chaperones identified so far in the ER are amongst the most abundant proteins in a cell, and even more so in the ER. Well-known and well-studied are the ER Hsp70 homolog BiP, the Hsp90 homolog Grp94, and the lectin chaperones calnexin (CNX) and calreticulin (CRT). Long-known folding enzymes include protein disulfide isomerase (PDI) and two other oxidoreductases: ERp72 and ERp57. More recently, ERp44 was identified in mammalian ER (Anelli et al. 2002), and the S. Cerevisiae genome revealed the existence of a total of 5 PDI family members in the yeast ER (Norgaard et al. 2001). Peptidyl-prolyl cis-trans isomerases (PPIases) constitute another class of enzymes present in the ER. They catalyse the isomerisation of peptide bonds between any amino acid and a proline in a polypeptide chain; their role in folding was mainly demonstrated in vitro. A very high number of PPIases were discovered in mammals, grouped in 3 families (cyclophilins, FK506 binding proteins and parvulins). Their functions reach beyond the assistance of newly synthesized proteins, being at the intersection between protein folding, signal transduction, trafficking, assembly and cell cycle regulation (Gothel and Marahiel 1999). Mechanistic details on some of the ER folding factors will be described below.

The question why particular newly synthesized proteins have preference for particular chaperones and folding enzymes has not been answered yet. Helenius and colleagues postulated that a hierarchy exists that depends on the position of the first glycan (Molinari and Helenius 2000). Important is the concept of redundancy. It exists everywhere in biology, and is prominent amongst chaperones and folding enzymes. If the favored chaperone or folding enzyme is not available (because of a defect, or because of competition) another ER folding factor takes over. This second folding factor may act through a different mechanism, but the end result, a high yield of properly folded protein, may be the same. Consequence of redundancy is the lower chance for misfolding, and at the 
same time an increased likelihood of competition. A cell's ER protein folding capacity therefore is likely to depend on the relative concentration of the various ER folding factors, as well as the nature of the proteins synthesized by that cell.

Viruses have evolved to exploit the cell's machineries, including those specialized in protein folding and quality control. Some viruses may have strategies to stop host protein synthesis altogether, but any virus will cause a shift in the balance in the ER. Viral proteins as a rule are much more abundant than endogenous newly synthesized proteins, such that they compete out other substrates for the ER folding factors. This is clearly illustrated when comparing the number of proteins co-immunoprecipitated with CNX from a noninfected cell lysate with the almost exclusive co-immunoprecipitation of X31 influenza virus hemagglutinin (HA) from an influenza infected cell lysate (Peterson et al. 1995), even though host protein synthesis is barely inhibited by this influenza strain.

The private chaperones are protein-specific factors that have evolved to assist the folding of protein (families) with atypical physical structures, or of proteins that exist in unusual situations or conditions. Collagen, for example, needs HSP47 to prevent premature fiber formation (Nagata 1996; Tasab et al. 2000) and RAP is considered to be specific for the LDL receptor family (Bu et al. 1995). Another example is the ER-resident molecule tapasin (Tpn) that is uniquely dedicated to tether class I molecules jointly with the chaperone calreticulin and the oxidoreductase ERp57 to TAP (Momburg and Tan 2002).

Whereas viral proteins are found abundantly associated with general folding factors, they are of course less likely to use such private chaperones, except when they would be virally encoded. On the other hand, we cannot exclude that viral proteins co-evolving with cells acquire(d) or exploit private chaperones. Up to now, the number of proteins (both viral and mammalian) for which biosynthesis is extensively studied is too low to allow general conclusions.

\section{Glycosylation and the CNX/CRT cycle}

Glycosylation has been shown to be important for protein folding, for protein stability, for immune evasion, and receptor usage. Most viral envelope proteins 
are glycoproteins, and they bind during folding to the lectin chaperones $\mathrm{CNX}$ and CRT (Parodi 2000). The process of N-linked glycosylation of secretory proteins is characterized by enzymatic reactions occurring on both sides of the ER membrane. Monosaccharides are added to the lipid intermediate dolichol pyrophosphate to make Man5GlcNac2-PP-Dol. Further elongation of the glycan chain depends on specific translocators called flippases (Helenius et al. 2002). Once "flipped" over to the ER lumenal side, the precursor is elongated to Glc3Man9GlcNac2-PP-Dol for which the oligosaccharyltransferase (OST) complex has optimal affinity. The enzyme transfers the oligosaccharide 14-mer en bloc to the nascent chain onto the consensus sequence Asn-X-Ser or Asn-X-Thr, with $\mathrm{X}$ being any amino acid except proline or aspartic acid.

General dogma says that glycans are crucial for proper glycoprotein folding. The reasons are understood to a large extent. Addition of a glycan adds a very large, strongly hydrophilic moiety to an otherwise perhaps more hydrophobic stretch of polypeptide. This will force this region of the protein away from the hydrophobic core of the protein. Another, direct, effect of glycosylation was found in peptides. A glycosylated peptide was shown to adopt a different conformation than the peptide without glycan (Imperiali and Rickert 1995), indicating a direct change of protein structure.

Studies with the glucosidase inhibitor n-butyl deoxynojirimycin (nBuDNJ) showed that the assembly of viral envelopes of viruses, such as e.g. HIV, was not affected. However, the virus could not enter the host cell because the rearrangment of the V1/V2 loops necessary to release gp120 from gp160 could not take place (Fischer et al. 1996) with gp41 unable to mediate fusion. This demonstrates the importance of correct glycosylation for viral proteins.

A more indirect role for glycans is their recognition by the lectin chaperones CNX and CRT. These proteins bind the carbohydrate chain of newly synthesized glycoproteins only when in the monoglucosylated form, reached after trimming of the N-linked glycan by action of ER glucosidases I and II. A prolonged interaction with CNX and CRT is established via reversible glucosylation of the N-linked glycan after glucosidase II has removed the important glucose. In this way, CNX and CRT bind and release substrate in cycles, giving prolonged chance to the 
substrate to fold correctly. The enzyme responsible for reglucosylation is the UDP-glucose:glycoprotein glucosyltransferase (GT) which behaves as a sensor for glycoprotein conformation (Parodi 2000; Ellgaard and Helenius 2001). The interaction with CNX and CRT exposes the folding protein to the associated cochaperone ERp57, a thiol oxidoreductase of the PDI family. During folding of viral glycoproteins, ERp57 has been shown to form transient intermolecular disulfide bonds with glycoprotein substrates bound to CNX and CRT (Molinari and Helenius 1999). The interaction between the substrate and one of the lectins seems to be required for the interaction with ERp57. Recently the threedimensional structure of the CRT P-domain and the CNX ectodomain have been solved (Ellgaard et al. 2001; Schrag et al. 2001; Ellgaard et al. 2002). A structural characterization of the binding site of ERp57 on CRT has been defined by NMR studies (Frickel et al. 2002). Whether ERp57 functions as a thiol oxidase and/or a disulfide isomerase still needs to be determined.

The lectin specificity of CNX and CRT is identical and they can bind to the same substrate, sometimes even simultaneously. VSV G protein, which has two Nlinked glycans, binds to CNX but not to CRT (Hammond et al. 1994). In contrast, Influenza HA associates with both lectins, albeit through different glycans: CRT binds preferentially with earlier folding intermediates and CNX associates also with a native monomeric form of the HA (Hebert et al. 1997). Such differences between CNX and CRT may reflect differential accessibility of the glycans in relation to the ER membrane, because CNX has a transmembrane anchor and CRT is a soluble protein (Danilczyk et al. 2000).

\section{Disulfide bond formation}

Many proteins that fold in the ER contain disulfide bonds, which are required for the protein's folding, stability and function. Disulfide bond formation starts during synthesis; during the folding process, both native and non-native disulfide bonds may form. One of the special features of the ER is its oxidising environment, similar to the extracellular space. The formation and isomerization of disulfide bonds is catalyzed by protein thiol-disulfide oxidoreductases in the ER. The acitvity of this class of proteins depends on a pair of cysteines arranged in a Cys$\mathrm{X}$-X-Cys motif which has become a hallmark of all proteins involved in the 
formation or breakage of disulfide bonds. PDI was one of the first identified proteins belonging to this class of enzymes and it can catalyse the formation, reduction or isomerisation of disulfide bonds, depending on the redox environment (Noiva 1999; Freedman et al. 2002).

The ER needs an oxidizing milieu to be able to support disulfide bond formation. Oxidized glutathione (GSSG) originally was thought to be responsible for generation and maintainance of the redox conditions in the ER, because of the higher ratio of oxidized to reduced glutathione in the ER (1:3) compared to the cytosol (1:60) (Hwang et al. 1992). Glutathione's assumed role, however, was completely changed by the identification of the Erolp protein in yeast (Frand and Kaiser 1998; Pollard et al. 1998) and Ero1-L $\square$ and $\square$ in mammalian cells (Cabibbo et al. 2000; Pagani et al. 2000). This protein binds to the main oxidoreductase PDI (Frand and Kaiser 1999; Benham et al. 2000;) and drives disulfide bond formation by maintaining PDI, and possibly other PDI family members such as Erp44 (Anelli et al. 2002), in the oxidised state necessary for disulfide transfer (Tu et al. 2000).

In yeast, electrons flow from the oxidised substrate via PDI to Erolp to FAD, with molecular oxygen as final electron acceptor (fig 1B insert) (Tu and Weissman 2002). Mammalian Ero1 has not been found to bind FAD (yet), and downstream electron acceptors still need to be identified. A second, Erol independent, pathway for disulfide formation is proposed to involve a small ER oxidase known as Erv2, a $22 \mathrm{kDa}$ protein that is non-covalently bound to FAD (Gerber et al. 2001; Sevier et al. 2001). The exact role of Erv2 in disulfide bond formation remains unclear still (Tu and Weissman 2002). In Vaccinia virus, three cytoplasmic thiol oxidoreductases were identified that comprise a complete pathway for disulfide bond formation for viral proteins in the relativeley reducing cytosol. Interestingly, the upstream component, E10R, is an Erv-like protein. These redox proteins are conserved in all poxviruses (Senkevich et al. 2002).

Disulfide bonds are usually highly conserved and critical for folding. They contribute to the stability of folding intermediates perhaps even more than to the stability of folded native proteins. Folding and disulfide bond formation coincide, 
meaning that folding can be followed by assaying formation and isomerization of disulfide bonds, for instance through pulse-chase experiments (Braakman and Herbert 1996). Almost everything we know about the role of cysteines and disulfide bonds in protein folding comes from studies on viral proteins. The redox conditions in the ER can be easily manipulated by addition of DTT in the culture medium (Alberini et al. 1990; Braakman et al. 1992): disulfide bond formation is inhibited but the effect is reversed when DTT is removed. This allowed postponing of disulfide bond formation until after synthesis: completely posttranslationally. Co-translational folding did increase efficiency of folding but turned out to be not essential, at least not in an intact cell where all ER factors are available.

Mutagenesis of cysteines involved in disulfide bonds has often been applied to study their influence on folding, maturation and intracellular transport. Often, deletion of one cysteine residue from a pair is more deleterious than removal of both because a single free cysteine is very reactive and can interfere with other cysteines normally involved in other disulfide bonds. The fact that highly variable viral envelope proteins contain completely conserved sets of cysteine residues and therefore most likely highly conserved disulfide bonds, demonstrates the importance of these covalent links for these proteins' functions.

\subsection{Quality control:}

\subsubsection{Retention in the ER}

During folding, proteins are subjected to a quality control machinery which allows exit from the ER only to proteins that have reached the correct three-dimensional structure. Misfolded and incompletely assembled proteins are retained in the ER and eventually degraded. The association of newly synthesised proteins with resident ER folding factors not only provides assistance during folding but it also reprensents the main mechanism for quality control, both at the co- and posttranslational level. Folding factors are indeed localized in the ER and association with them results in retention of the folding substrate in the ER until proper folding is reached (Ellgaard et al. 1999). Some incompletely assembled substrates 
can also be retained in the ER through exposure of a more specific feature. An example of this is thiol-mediated retention, which was observed first for the immunoglobulin IgM (Sitia et al. 1990). During oligomerization, the exposure of a cysteine residue in the tailpiece (cys 575), involved in an interchain disulfide bond in the polymer, is a signal for ER retention as well as for degradation (Fra et al. 1993).

For glycoproteins, the CNX/CRT cycle is a common quality control system, which allows the protein multiple chances to fold correctly. The exit of certain glycoproteins from the ER to the Golgi complex is assisted by another membranebound lectin, ERGIC-53, which recognizes mannose residues (Hauri et al. 2000). In addition to cellular factors, proper folding of viral proteins may require complex interactions with neighbouring viral proteins (see section 6.1.2; Braakman and van Anken 2000). Many viral envelopes consist of highly ordered scaffolds of strongly interacting structural proteins. These regular interactions may require more stringent conformations for the structural proteins, resulting in perhaps a more stringent quality control compared to the quality control of cellular protein $\mathrm{s}$. It is not easy to conclude this from available data, because of the limited set of proteins for which folding and quality control has been studied. Actually, viral glycoproteins frequently serve as model proteins for studies on protein folding in the ER (Doms et al. 1993). Not only for viral but also for cellular proteins, large variations exist concerning retention in the ER: where of CFTR even the wild-type protein is degraded for more than 50\% (Ward and Kopito 1994), mutants of the LDL-receptor often leave the ER to reach the plasma membrane (Hobbs et al. 1992).

\subsubsection{Degradation and the unfolded protein response}

Prolonged retention of misfolded and incompletely folded proteins in the ER leads to their degradation (ER associated degradation or ERAD) (Brodsky and McCracken 1999). This process involves the translocon, the cytosolic proteasome and ER chaperones such as CNX , BiP and PDI (Zhang et al. 2001; Molinari et al. 2002). The substrate to be degraded is retrotranslocated through the Sec61 channel into the cytosol, deglycosylated by a cytosolic N-glycanase and, often 
polyubiquitinated before proteasomal degradation (Tsai et al. 2002). The criteria that determine how proteins are identified and sent to the degradation machinery are not completely understood. In eukaryotes, misfolded proteins are recognized by the enzyme mannosidase I, which removes the terminal mannose residue, producing the sugar moiety Man8GlcNAc2. The $\mathrm{Man}_{8}$-species (or $\mathrm{Glc}_{1} \mathrm{Man}_{8}$ for that matter) is recognized by a lectin, which is related to $\square$-mannosidase but lacks enzymatic activity (called Htm1 or Mnl1 in yeast, and EDEM in mammals) (Jakob et al. 2001; Nakatsukasa et al. 2001). The lectin is thought to target the protein to the retro-translocation pathway. Inhibition of mannosidase I, or deletion of the lectin, stabilizes some misfolded glycoproteins. Which mechanism targets nonglycosylated proteins is still not known.

Together the CNX/CRT cycle and the ER mannosidase allow incorrectly folded proteins multiple chances over prolonged periods of time to acquire the correct conformation. To handle the accumulation of incorrectly folded proteins in the ER, however, a signalling machinery has evolved that communicates to the nucleus that protein expression needs to be modulated to alleviate cellular stress, referred to as the unfolded protein response (UPR) (Ma and Hendershot 2001; Patil and Walter 2001; Harding et al. 2002). The result is an upregulation of genes encoding ER folding factors. Studies in S.cerevisiae have shown that the processes of UPR and ERAD are functionally linked to each other (Friedlander et al. 2000; Travers et al. 2000). In this way, cells can at the same time increase ER protein folding capacity and reduce ER protein folding load.

It is not surprising that upon viral infection, the heavy load of viral protein that needs to be handled leads to an upregulation of ER. Also not suprising is the cellular stress a viral infection may cause. Question is whether either the high viral activity within the cell or the accumulation of viral proteins induces stress. Two examples of viruses inducing UPR are the Hepatitis C virus (HCV) and Japanese encephalitis virus (JEV). HCV replication induces ER stress with the activation of UPR through transcription factor ATF6 and increased transcript levels of BiP (Tardif et al. 2002). During Japanese encephalitis virus (JEV) infection, the lumen of the ER rapidly accumulates substantial amounts of viral 
proteins that triggers the UPR with the activation of CHOP/GADD153, a distinctive transcription factor often induced by UPR (Su et al. 2002).

\subsection{How viruses evolve to fool the host}

For a productive infection, viruses need to reproduce into as many particles as possible. They can subvert the host cell translation apparatus, affecting several steps in the process of protein synthesis: degradation of host mRNA, competition for the host translation apparatus, changing the specificity of the host translation apparatus. In some cases, cellular protein synthesis is almost completely shut-off, but this becomes rapidly toxic to the cell. A more limited inhibition of cellular functions usually is preferred to allow a long and productive infection.

When virus production is ensured in an infected cell, the next problem is to escape the organism's bodyguards. The immune system evolved to counter attack viral infections, but viruses have the possibility to evolve more rapidly and they have developed many tricks to evade the immune survaillance. The immune system is mainly based on humoral and cellular responses and both are targets for viral evasion.

The humoral response is based on recognition of viruses and viral proteins in solution. Envelope glycoproteins, being on the viral surface, are very immunogenic. Mammalian viruses therefore are subjected to tremendous selective pressures to continually change their molecular profiles. In many cases, natural selection produces viral strains that vary considerably in the antigenic regions of their spike proteins. The main strategy to mask the envelope glycoproteins from the immune system is by antigenic variation. RNA viruses are far more susceptible to genetic variation than DNA viruses. Three main mechanisms have been described: 1) point mutations (antigenic drift), 2) recombination, and 3) reassortment (antigenic shift).

1) RNA viruseses lack a proof reader for replication, allowing the virus to mutate rapidly and frequently. RNA polymerases are at least 1,000-10,000 times more 
prone to error than DNA polymerases, which is responsible for the far higher mutation rates for RNA viruses. Hot spots for mutation often occur in the viral genome, coinciding in particular with antigenic sites recognized by virusneutralizing antibodies. Influenza and HIV virus are two well-characterized examples of viruses that use this strategy.

Another attempt to mask the viral envelope proteins to the humoral immune system is by using host glycosylation as a means to cover or change potential antigenic epitopes. Most envelope glycoproteins are indeed heavily glycosylated and glycosylation sites are easily added or deleted during evolution through antigenic drift. HIV is a clear example of a virus with a highly glycosylated envelope protein ( $\sim 30$ glycans) where for most glycans not the position in the protein but rather the gross number of glycans is conserved. For folding of a glycoprotein, the exact position is less important than the presence per se of a glycan in that region of the polypeptide chain. Moving a glycan over the surface of the protein often does not affect folding and maturation, but does effect shielding of antigenic sites, or disappearance of an antigenic epitope if the glycan was part of that epitope. It is possible that the virus maintains the number of glycans because they indeed help the virus to pass through cells and body unobserved.

2) Viruses can undergo recombination, during which genetic material is exchanged with related viral or cellular sequences through cutting and splicing of nucleic acids. This leads to a sudden change of the expressed proteins. This feature is prominent in virus families that contain positively stranded RNA, such as the Picornaviridae and the Coronaviridae.

3) Finally, reassortment of the individual pieces of RNA may occur during a mixed infection. For Influenza virus, which doesn't show recombination as defined in 2), this frequently occurring antigenic shift is sometimes called recombination. Reassortment, however, leads to the production of a virus encoding proteins of different origin. This is evident in viruses in which the mRNA genome is present in multiple different segments which can be exchanged without any need to cut and splice nucleic acids. Other virus families with 
multiple genome segments include the Rotaviridae and the Bunyaviridae.This mechanism is postulated to account for the major antigenic shift of influenza A and is responsible for epidemic outbreaks of this virus.

Using a cellular response, the immune system not only recognizes virions in solution but also infected cells. Like cellular proteins, intracellular viral proteins are degraded and the resulting peptides are presented by specific molecules on the cell surface. The $\mathrm{T}$ cell receptor, present on $\mathrm{T}$ cells, recognizes the antigen presenting molecules, MHC class I and II associated with peptide. Recognition of a foreign antigen will result, through different mechanims, in cell death. Almost every step in the antigen presentation pathway (both by MCH I and II) has been taken as a target for viral subversion (for complete reviews, see Tortorella et al. 2000; Vossen et al. 2002).

We here will describe only the viral products that interfere with mechanisms related to the secretory pathway. Most examples involve the MHC class I presentation route. First, peptides are produced through proteasomal degradation of viral proteins. Some viral products (EBNA 1 of Epstein-Barr virus, for example) avoid their own degradation by insertion of Gly-Ala repeats in their sequence. These repeats work as cis-acting inhibitor of the proteasomal proteolytic enzymes (Levitskaya et al. 1997; Leonchiks et al. 2002). Once peptides are formed, they need to be transferred into the ER through a channel, the MHC-encoded peptide transporter TAP. The ER resident protein Tapasin is associated with TAP. Some viruses produce a protein that interacts with the cytosolic part of TAP (such as Herpes Virus Simplex ICP47) (York et al. 1994), others, such as Human Cytomagalovirus US6, interact with TAP on the ER lumenal face (Hengel et al. 1997). Binding of US6 to TAP stabilizes a conformation of TAP that is unable to bind ATP and hence cannot translocate peptide (Hewitt et al. 2001). US6 itself does not contain a retention signal to stably reside in the ER, but was suggested to bind to calnexin, through which it would be retained in the ER (Hengel et al. 1997).

After assembly of MCH I with peptide, the complex normally would be transported to the cell surface, but many virus products are involved in its 
intracellular retention. MHC class I can be retained in the ER for example by binding with the adenoviral product E3-19k (Cox et al. 1991), which in addition bind to TAP and act as a competitive inhibitor of tapasin (Bennett et al. 1999). Retention can also occur in the ERGIC/cis-Golgi compartment by binding with the murine Cytomegalovirus (MCMV) protein gp40 (m152), which has a retention signal (Ziegler et al. 1997). Two other proteins, gp48 (m06) and gp34 (m04), made by the same MCMV, interfere with MHC-I molecules. Gp48 directs class I molecules to the lysosomes whereas gp34 interacts with MHC-I in the ER; in addition, the gp34/class I complex is found on the cell surface. The exact function of gp34 is not completely understood. It may function to silence the NK response and/ or alter the interaction with CD8+ T cells (Holtappels et al. 2000).

HCMV uses other tricks to prevent MHC-I from reaching the plasma membrane: the US3 protein retains MHC-I in the ER with a still unknown mechanism. The same US3 can also bind to MHC-II reducing their association with invariant chain (Ii) (Hegde et al. 2002). Another HCMV gene product, US2 is implied in degradation of two important proteins of MHC-II (Tomazin et al. 1999) and, together with US11, efficiently direct class I molecules for proteasomal degradation with a half time of less than 1 minute. These viral studies at the same time showed the first example of what turned out to be a more general process, where misfolded proteins in the ER need dislocation into the cytosol to be degraded by the proteasome (Wiertz et al. 1996; van der Wal et al. 2002).

HIV virus interferes with MHC-I through two gene products: Nef and Vpu. Nef accelerates endocytosis of MHC-I (Schwartz et al. 1996) and CD4 (Rhee and Marsh 1994). The MHC-I-nef complex accumulates in the TGN and is then delivered to lysosomes. The mechanism of endocytosis for MHC-I and CD4 is different. Endocytosis of CD4 involves clathrin coated pits (Piguet et al. 1998; Williams et al. 2002) whereas MHC-I was recently connected to the ARF6 endocytic pathway (Blagoveshchenskaya et al. 2002). Another HIV protein, Vpu, induces destabilization of newly synthesised MHC-I and induces proteasomal degradation of newly synthesised CD4 by phosphorylation of the protein on two specific residues (Fujita et al. 1997; Kerkau et al. 1997; Paul and Jabbar 1997). 
Viruses evolved into enormously efficient infectious agents that keep their host alive for as long as possible to allow production of the maximum amount of progeny virus. The study of every strategy viruses developed to survive, increases our knowledge on cellular and immunological processes and will improve chances to develop immunotherapies for treatment of viral infections.

\author{
Abbreviations: \\ CNX calnexin \\ CRT calreticulin \\ ER endoplasmic reticulum \\ GT UDP-glucose:glycoprotein glucosyltransferase \\ HA Influenza Haemagglutinin \\ UPR unfolded protein response \\ MHC Major Histocompatibility Class
}

\title{
Acknoledgments
}

We thank Marije Liscalijet and Eelco van Anken for critical reading of the manuscript and the audio visual department, especially Aloys Lurvink, for assistance with the figure.

Alberini CM, P Bet, C Milstein and R Sitia (1990). Secretion of immunoglobulin $\mathrm{M}$ assembly intermediates in the presence of reducing agents. Nature 347(6292): 485-7.

Andersson H, BU Barth, M Ekstrom and H Garoff (1997). Oligomerizationdependent folding of the membrane fusion protein of Semliki Forest virus. J Virol 71(12): 9654-63.

Anelli T, M Alessio, A Mezghrani, T Simmen, F Talamo, A Bachi and R Sitia (2002). ERp44, a novel endoplasmic reticulum folding assistant of the thioredoxin family. EMBO J 21(4): 835-44.

Anfinsen CB (1973). Principles that govern the folding of protein chains. Science 181(96): 223-30.

Benham AM, A Cabibbo, A Fassio, N Bulleid, R Sitia and I Braakman (2000). The CXXCXXC motif determines the folding, structure and stability of human Ero1-L૫. EMBO J 19(17): 4493-502. 
Bennett EM, JR Bennink, JW Yewdell and FM Brodsky (1999). Cutting edge: adenovirus E19 has two mechanisms for affecting class I MHC expression. J Immunol 162(9): 5049-52.

Berman PW, WM Nunes and OK Haffar (1988). Expression of membraneassociated and secreted variants of gp160 of human immunodeficiency virus type 1 in vitro and in continuous cell lines. J Virol 62(9): 3135-42. Blagoveshchenskaya AD, L Thomas, SF Feliciangeli, CH Hung and G Thomas (2002). HIV-1 Nef Downregulates MHC-I by a PACS-1- and PI3KRegulated ARF6 Endocytic Pathway. Cell 111(6): 853-66.

Braakman I, J Helenius and A Helenius (1992). Manipulating disulfide bond formation and protein folding in the endoplasmic reticulum. EMBO J 11(5): 1717-22.

Braakman, I., D. Hebert. Disulfide (-SS-) bond formation overview. In: Current Protocols in Protein Science, Chapter 14.1 (eds. J. Coligan, B. Dunn, H. Ploegh, D. Speicher, P. Wingfield; John Wiley and Sons, Inc, New York, 1996): 14.1.1-14.1.15.

Braakman I and E van Anken (2000). Folding of viral envelope glycoproteins in the endoplasmic reticulum. Traffic 1(7): 533-9.

Brodsky JL and AA McCracken (1999). ER protein quality control and proteasome-mediated protein degradation. Semin Cell Dev Biol 10(5): 507-13.

Bu G, HJ Geuze, GJ Strous and AL Schwartz (1995). 39 kDa receptor-associated protein is an ER resident protein and molecular chaperone for LDL receptor-related protein. EMBO J 14(10): 2269-80.

Cabibbo A, M Pagani, M Fabbri, M Rocchi, MR Farmery, NJ Bulleid and R Sitia (2000). ERO1-L, a human protein that favors disulfide bond formation in the endoplasmic reticulum. J Biol Chem 275(7): 4827-33.

Carleton M, H Lee, M Mulvey and DT Brown (1997). Role of glycoprotein PE2 in formation and maturation of the Sindbis virus spike. J Virol 71(2): $1558-66$

Cocquerel L, JC Meunier, A Op de Beeck, D Bonte, C Wychowski and J Dubuisson (2001). Coexpression of hepatitis C virus envelope proteins E1 and E2 in cis improves the stability of membrane insertion of E2. J Gen Virol 82(Pt 7): 1629-35. 
Cox JH, JR Bennink and JW Yewdell (1991). Retention of adenovirus E19 glycoprotein in the endoplasmic reticulum is essential to its ability to block antigen presentation. J Exp Med 174(6): 1629-37.

Danilczyk UG, MF Cohen-Doyle and DB Williams (2000). Functional relationship between calreticulin, calnexin, and the endoplasmic reticulum luminal domain of calnexin. J Biol Chem 275(17): 13089-97.

Doms RW, RA Lamb, JK Rose and A Helenius (1993). Folding and assembly of viral membrane proteins. Virology 193(2): 545-62.

Duvet S, A Op De Beeck, L Cocquerel, C Wychowski, R Cacan and J Dubuisson (2002). Glycosylation of the hepatitis C virus envelope protein E1 occurs posttranslationally in a mannosylphosphoryldolichol-deficient $\mathrm{CHO}$ mutant cell line. Glycobiology 12(2): 95-101.

Ellgaard L, P Bettendorff, D Braun, T Herrmann, F Fiorito, I Jelesarov, P Guntert, A Helenius and K Wuthrich (2002). NMR structures of 36 and 73-residue fragments of the calreticulin P-domain. J Mol Biol 322(4): 773-84.

Ellgaard L and A Helenius (2001). ER quality control: towards an understanding at the molecular level. Curr Opin Cell Biol 13(4): 431-7.

Ellgaard L, M Molinari and A Helenius (1999). Setting the standards: quality control in the secretory pathway. Science 286(5446): 1882-8.

Ellgaard L, R Riek, D Braun, T Herrmann, A Helenius and K Wuthrich (2001). Three-dimensional structure topology of the calreticulin P-domain based on NMR assignment. FEBS Lett 488(1-2): 69-73.

Ellis RJ (2001). Macromolecular crowding: an important but neglected aspect of the intracellular environment. Curr Opin Struct Biol 11(1): 114-9.

Fischer PB, GB Karlsson, TD Butters, RA Dwek and FM Platt (1996). Nbutyldeoxynojirimycin-mediated inhibition of human immunodeficiency virus entry correlates with changes in antibody recognition of the V1/V2 region of gp120. J Virol 70(10): 7143-52.

Fischer WB and MS Sansom (2002). Viral ion channels: structure and function. Biochim Biophys Acta 1561(1): 27-45.

Fra AM, C Fagioli, D Finazzi, R Sitia and CM Alberini (1993). Quality control of ER synthesized proteins: an exposed thiol group as a three-way switch mediating assembly, retention and degradation. EMBO J 12(12): 4755-61. 
Frand AR and CA Kaiser (1998). The ERO1 gene of yeast is required for oxidation of protein dithiols in the endoplasmic reticulum. Mol Cell 1(2): 161-70.

Frand AR and CA Kaiser (1999). Erolp oxidizes protein disulfide isomerase in a pathway for disulfide bond formation in the endoplasmic reticulum. Mol Cell 4(4): 469-77.

Freedman RB, P Klappa and LW Ruddock (2002). Protein disulfide isomerases exploit synergy between catalytic and specific binding domains. EMBO Rep 3(2): 136-40.

Frickel EM, R Riek, I Jelesarov, A Helenius, K Wuthrich and L Ellgaard (2002). TROSY-NMR reveals interaction between ERp57 and the tip of the calreticulin P-domain. Proc Natl Acad Sci U S A 99(4): 1954-9.

Friedlander R, E Jarosch, J Urban, C Volkwein and T Sommer (2000). A regulatory link between ER-associated protein degradation and the unfolded-protein response. Nat Cell Biol 2(7): 379-84.

Fujita K, S Omura and J Silver (1997). Rapid degradation of CD4 in cells expressing human immunodeficiency virus type $1 \mathrm{Env}$ and $\mathrm{Vpu}$ is blocked by proteasome inhibitors. J Gen Virol 78(Pt 3): 619-25.

Gerber J, U Muhlenhoff, G Hofhaus, R Lill and T Lisowsky (2001). Yeast ERV2p is the first microsomal FAD-linked sulfhydryl oxidase of the Erv1p/Alrp protein family. J Biol Chem 276(26): 23486-91.

Gorlich D and TA Rapoport (1993). Protein translocation into proteoliposomes reconstituted from purified components of the endoplasmic reticulum membrane. Cell 75(4): 615-30.

Gothel SF and MA Marahiel (1999). Peptidyl-prolyl cis-trans isomerases, a superfamily of ubiquitous folding catalysts. Cell Mol Life Sci 55(3): 42336.

Haigh NG and AE Johnson (2002). A new role for BiP: closing the aqueous translocon pore during protein integration into the ER membrane. J Cell Biol 156(2): 261-70.

Hammond C, I Braakman and A Helenius (1994). Role of N-linked oligosaccharide recognition, glucose trimming, and calnexin in glycoprotein folding and quality control. Proc Natl Acad Sci U S A 91(3): 913-7. 
Harding HP, M Calfon, F Urano, I Novoa and D Ron (2002). Transcriptional and translational control in the Mammalian unfolded protein response. Annu Rev Cell Dev Biol 18: 575-99.

Hartmann E, D Gorlich, S Kostka, A Otto, R Kraft, S Knespel, E Burger, TA Rapoport and S Prehn (1993). A tetrameric complex of membrane proteins in the endoplasmic reticulum. Eur J Biochem 214(2): 375-81.

Hauri H, C Appenzeller, F Kuhn and O Nufer (2000). Lectins and traffic in the secretory pathway. FEBS Lett 476(1-2): 32-7.

Hebert DN, JX Zhang, W Chen, B Foellmer and A Helenius (1997). The number and location of glycans on influenza hemagglutinin determine folding and association with calnexin and calreticulin. J Cell Biol 139(3): 613-23.

Hegde NR, RA Tomazin, TW Wisner, C Dunn, JM Boname, DM Lewinsohn and DC Johnson (2002). Inhibition of HLA-DR assembly, transport, and loading by human cytomegalovirus glycoprotein US3: a novel mechanism for evading major histocompatibility complex class II antigen presentation. J Virol 76(21): 10929-41.

Hegde RS, S Voigt, TA Rapoport and VR Lingappa (1998). TRAM regulates the exposure of nascent secretory proteins to the cytosol during translocation into the endoplasmic reticulum. Cell 92(5): 621-31.

Helenius J, DT Ng, CL Marolda, P Walter, MA Valvano and M Aebi (2002). Translocation of lipid-linked oligosaccharides across the ER membrane requires Rft1 protein. Nature 415(6870): 447-50.

Hengel H, JO Koopmann, T Flohr, W Muranyi, E Goulmy, GJ Hammerling, UH Koszinowski and F Momburg (1997). A viral ER-resident glycoprotein inactivates the MHC-encoded peptide transporter. Immunity 6(5): 623-32. Hesketh JE and IF Pryme (1991). Interaction between mRNA, ribosomes and the cytoskeleton. Biochem J 277(Pt 1): 1-10.

Hewitt EW, SS Gupta and PJ Lehner (2001). The human cytomegalovirus gene product US6 inhibits ATP binding by TAP. EMBO J 20(3): 387-96.

Hobbs HH, MS Brown and JL Goldstein (1992). Molecular genetics of the LDL receptor gene in familial hypercholesterolemia. Hum Mutat 1(6): 445-66.

Holtappels R, D Thomas, J Podlech, G Geginat, HP Steffens and MJ Reddehase (2000). The putative natural killer decoy early gene m04 (gp34) of murine 
cytomegalovirus encodes an antigenic peptide recognized by protective antiviral CD8 T cells. J Virol 74(4): 1871-84.

Hwang C, AJ Sinskey and HF Lodish (1992). Oxidized redox state of glutathione in the endoplasmic reticulum. Science 257(5076): 1496-502.

Imperiali B and KW Rickert (1995). Conformational implications of asparaginelinked glycosylation. Proc Natl Acad Sci U S A 92(1): 97-101.

Jakob CA, D Bodmer, U Spirig, P Battig, A Marcil, D Dignard, JJ Bergeron, DY Thomas and M Aebi (2001). Htm1p, a mannosidase-like protein, is involved in glycoprotein degradation in yeast. EMBO Rep 2(5): 423-30.

Johnson AE and MA van Waes (1999). The translocon: a dynamic gateway at the ER membrane. Annu Rev Cell Dev Biol 15: 799-842.

Kerkau T, I Bacik, JR Bennink, JW Yewdell, T Hunig, A Schimpl and U Schubert (1997). The human immunodeficiency virus type 1 (HIV-1) Vpu protein interferes with an early step in the biosynthesis of major histocompatibility complex (MHC) class I molecules. J Exp Med 185(7): 1295-305.

Land A, D Zonneveld and I Braakman (2003). Folding of HIV-1 envelope glycoprotein involves extensive isomerization of disulfide bonds and conformation-dependent signal peptide cleavage. FASEB J in press.

Leonchiks A, V Stavropoulou, A Sharipo and MG Masucci (2002). Inhibition of ubiquitin-dependent proteolysis by a synthetic glycine-alanine repeat peptide that mimics an inhibitory viral sequence. FEBS Lett 522(1-3): 938.

Levitskaya J, A Sharipo, A Leonchiks, A Ciechanover and MG Masucci (1997). Inhibition of ubiquitin/proteasome-dependent protein degradation by the Gly-Ala repeat domain of the Epstein-Barr virus nuclear antigen 1. Proc Natl Acad Sci U S A 94(23): 12616-21.

Li Y, L Luo, DY Thomas and CY Kang (1994). Control of expression, glycosylation, and secretion of HIV-1 gp120 by homologous and heterologous signal sequences. Virology 204(1): 266-78.

Lorenz IC, SL Allison, FX Heinz and A Helenius (2002). Folding and dimerization of tick-borne encephalitis virus envelope proteins prM and $\mathrm{E}$ in the endoplasmic reticulum. J Virol 76(11): 5480-91.

Ma Y and LM Hendershot (2001). The unfolding tale of the unfolded protein response. Cell 107(7): 827-30. 
Matlack KE, W Mothes and TA Rapoport (1998). Protein translocation: tunnel vision. Cell 92(3): 381-90.

Melancon P and H Garoff (1987). Processing of the Semliki Forest virus structural polyprotein: role of the capsid protease. J Virol 61(5): 1301-9.

Merola M, M Brazzoli, F Cocchiarella, JM Heile, A Helenius, AJ Weiner, M Houghton and S Abrignani (2001). Folding of hepatitis C virus E1 glycoprotein in a cell-free system. J Virol 75(22): 11205-17.

Mezghrani A, A Fassio, A Benham, T Simmen, I Braakman and R Sitia (2001). Manipulation of oxidative protein folding and PDI redox state in mammalian cells. EMBO J 20(22): 6288-96.

Michalak JP, C Wychowski, A Choukhi, JC Meunier, S Ung, CM Rice and J Dubuisson (1997). Characterization of truncated forms of hepatitis C virus glycoproteins. J Gen Virol 78(Pt 9): 2299-306.

Molinari M, C Galli, V Piccaluga, M Pieren and P Paganetti (2002). Sequential assistance of molecular chaperones and transient formation of covalent complexes during protein degradation from the ER. J Cell Biol 158(2): 247-57.

Molinari M and A Helenius (1999). Glycoproteins form mixed disulphides with oxidoreductases during folding in living cells. Nature 402(6757): 90-3.

Molinari M and A Helenius (2000). Chaperone selection during glycoprotein translocation into the endoplasmic reticulum. Science 288(5464): 331-3.

Momburg F and P Tan (2002). Tapasin-the keystone of the loading complex optimizing peptide binding by MHC class I molecules in the endoplasmic reticulum. Mol Immunol 39(3-4): 217-33.

Nagata K (1996). Hsp47: a collagen-specific molecular chaperone. Trends Biochem Sci 21(1): 22-6.

Nakatsukasa K, S Nishikawa, N Hosokawa, K Nagata and T Endo (2001). Mnl1p, an alpha -mannosidase-like protein in yeast Saccharomyces cerevisiae, is required for endoplasmic reticulum-associated degradation of glycoproteins. J Biol Chem 276(12): 8635-8.

Noiva R (1999). Protein disulfide isomerase: the multifunctional redox chaperone of the endoplasmic reticulum. Semin Cell Dev Biol 10(5): 481-93. 
Norgaard P, V Westphal, C Tachibana, L Alsoe, B Holst and JR Winther (2001). Functional differences in yeast protein disulfide isomerases. J Cell Biol 152(3): 553-62.

Pagani M, M Fabbri, C Benedetti, A Fassio, S Pilati, NJ Bulleid, A Cabibbo and R Sitia (2000). Endoplasmic reticulum oxidoreductin 1-lbeta (ERO1Lbeta), a human gene induced in the course of the unfolded protein response. J Biol Chem 275(31): 23685-92.

Parodi AJ (2000). Protein glucosylation and its role in protein folding. Annu Rev Biochem 69: 69-93.

Patel J, AH Patel and J McLauchlan (2001). The transmembrane domain of the hepatitis $\mathrm{C}$ virus $\mathrm{E} 2$ glycoprotein is required for correct folding of the E1 glycoprotein and native complex formation. Virology 279(1): 58-68.

Patil C and P Walter (2001). Intracellular signaling from the endoplasmic reticulum to the nucleus: the unfolded protein response in yeast and mammals. Curr Opin Cell Biol 13(3): 349-55.

Paul M and MA Jabbar (1997). Phosphorylation of both phosphoacceptor sites in the HIV-1 Vpu cytoplasmic domain is essential for Vpu-mediated ER degradation of CD4. Virology 232(1): 207-16.

Peterson JR, A Ora, PN Van and A Helenius (1995). Transient, lectin-like association of calreticulin with folding intermediates of cellular and viral glycoproteins. Mol Biol Cell 6(9): 1173-84.

Piguet V, YL Chen, A Mangasarian, M Foti, JL Carpentier and D Trono (1998). Mechanism of Nef-induced CD4 endocytosis: Nef connects CD4 with the mu chain of adaptor complexes. EMBO J 17(9): 2472-81.

Pollard MG, KJ Travers and JS Weissman (1998). Ero1p: a novel and ubiquitous protein with an essential role in oxidative protein folding in the endoplasmic reticulum. Mol Cell 1(2): 171-82.

Rehm A, P Stern, HL Ploegh and D Tortorella (2001). Signal peptide cleavage of a type I membrane protein, HCMV US11, is dependent on its membrane anchor. EMBO J 20(7): 1573-82.

Rhee SS and JW Marsh (1994). Human immunodeficiency virus type 1 Nefinduced down-modulation of CD4 is due to rapid internalization and degradation of surface CD4. J Virol 68(8): 5156-63. 
Schrag JD, JJ Bergeron, Y Li, S Borisova, M Hahn, DY Thomas and M Cygler (2001). The Structure of calnexin, an ER chaperone involved in quality control of protein folding. Mol Cell 8(3): 633-44.

Schwartz O, V Marechal, S Le Gall, F Lemonnier and JM Heard (1996).

Endocytosis of major histocompatibility complex class I molecules is induced by the HIV-1 Nef protein. Nat Med 2(3): 338-42.

Senkevich TG, CL White, EV Koonin and B Moss (2002). Complete pathway for protein disulfide bond formation encoded by poxviruses. Proc Natl Acad Sci U S A 99(10): 6667-72.

Sevier CS, JW Cuozzo, A Vala, F Aslund and CA Kaiser (2001). A flavoprotein oxidase defines a new endoplasmic reticulum pathway for biosynthetic disulphide bond formation. Nat Cell Biol 3(10): 874-82.

Sitia R, M Neuberger, C Alberini, P Bet, A Fra, C Valetti, G Williams and C Milstein (1990). Developmental regulation of IgM secretion: the role of the carboxy-terminal cysteine. Cell 60(5): 781-90.

Su HL, CL Liao and YL Lin (2002). Japanese encephalitis virus infection initiates endoplasmic reticulum stress and an unfolded protein response. J Virol 76(9): 4162-71.

Tardif KD, K Mori and A Siddiqui (2002). Hepatitis C virus subgenomic replicons induce endoplasmic reticulum stress activating an intracellular signaling pathway. J Virol 76(15): 7453-9.

Tasab M, MR Batten and NJ Bulleid (2000). Hsp47: a molecular chaperone that interacts with and stabilizes correctly-folded procollagen. EMBO J 19(10): 2204-11.

Tomazin R, J Boname, NR Hegde, DM Lewinsohn, Y Altschuler, TR Jones, P Cresswell, JA Nelson, SR Riddell and DC Johnson (1999).

Cytomegalovirus US2 destroys two components of the MHC class II pathway, preventing recognition by CD4+ T cells. Nat Med 5(9): 1039-43.

Tortorella D, BE Gewurz, MH Furman, DJ Schust and HL Ploegh (2000). Viral subversion of the immune system. Annu Rev Immunol 18: 861-926.

Travers KJ, CK Patil, L Wodicka, DJ Lockhart, JS Weissman and P Walter (2000). Functional and genomic analyses reveal an essential coordination between the unfolded protein response and ER-associated degradation. Cell 101(3): 249-58. 
Tsai B, Y Ye and TA Rapoport (2002). Retro-translocation of proteins from the endoplasmic reticulum into the cytosol. Nat Rev Mol Cell Biol 3(4): 24655.

Tu BP, SC Ho-Schleyer, KJ Travers and JS Weissman (2000). Biochemical basis of oxidative protein folding in the endoplasmic reticulum. Science 290(5496): 1571-4.

Tu BP and JS Weissman (2002). The FAD- and O(2)-dependent reaction cycle of Ero1-mediated oxidative protein folding in the endoplasmic reticulum. Mol Cell 10(5): 983-94.

van der Wal FJ, M Kikkert and E Wiertz (2002). The HCMV gene products US2 and US11 target MHC class I molecules for degradation in the cytosol. Curr Top Microbiol Immunol 269: 37-55.

Vossen MT, EM Westerhout, C Soderberg-Naucler and EJ Wiertz (2002). Viral immune evasion: a masterpiece of evolution. Immunogenetics 54(8): 52742.

Wang L and B Dobberstein (1999). Oligomeric complexes involved in translocation of proteins across the membrane of the endoplasmic reticulum. FEBS Lett 457(3): 316-22.

Ward CL and RR Kopito (1994). Intracellular turnover of cystic fibrosis transmembrane conductance regulator. Inefficient processing and rapid degradation of wild-type and mutant proteins. J Biol Chem 269(41): 25710-8.

Wiertz EJ, TR Jones, L Sun, M Bogyo, HJ Geuze and HL Ploegh (1996). The human cytomegalovirus US11 gene product dislocates MHC class I heavy chains from the endoplasmic reticulum to the cytosol. Cell 84(5): 769-79.

Williams M, JF Roeth, MR Kasper, RI Fleis, CG Przybycin and KL Collins (2002). Direct binding of human immunodeficiency virus type $1 \mathrm{Nef}$ to the major histocompatibility complex class I (MHC-I) cytoplasmic tail disrupts MHC-I trafficking. J Virol 76(23): 12173-84.

Wolin SL and P Walter (1988). Ribosome pausing and stacking during translation of a eukaryotic mRNA. EMBO J 7(11): 3559-69.

York IA, C Roop, DW Andrews, SR Riddell, FL Graham and DC Johnson (1994). A cytosolic herpes simplex virus protein inhibits antigen presentation to CD8+ T lymphocytes. Cell 77(4): 525-35. 
Zhang Y, G Nijbroek, ML Sullivan, AA McCracken, SC Watkins, S Michaelis and JL Brodsky (2001). Hsp70 molecular chaperone facilitates endoplasmic reticulum-associated protein degradation of cystic fibrosis transmembrane conductance regulator in yeast. Mol Biol Cell 12(5): 130314.

Ziegler H, R Thale, P Lucin, W Muranyi, T Flohr, H Hengel, H Farrell, W Rawlinson and UH Koszinowski (1997). A mouse cytomegalovirus glycoprotein retains MHC class I complexes in the ERGIC/cis-Golgi compartments. Immunity 6(1): 57-66. 


\section{FIG.1 Inside the crowded ER}

A. Model for ER crowding: in the lumen of the ER, newly synthesised proteins are welcomed in a very crowded environment. Proteins are depicted according to relative size and shape.

B. Venn diagram showing the overlapping functions of some folding factors. The insert shows one of the known processes in protein folding: the formation of disulfide bonds in a nascent protein. The final electron acceptors (gray box) were shown for yeast but are still unclear for mammalian cells. 